Ophthalmologe 2010 - 107:628-635

DOI 10.1007/s00347-010-2176-x

Online publiziert: 10. Juni 2010

(c) Springer-Verlag 2010
J.G. Garweg ${ }^{1} \cdot$ A. Wenzel ${ }^{2}$

${ }^{1}$ Swiss Eye Institute, Berner Augenklinik am Lindenhofspital, Universität Bern

2 Universität Zürich

\title{
Diabetische Makulopathie und Retinopathie
}

\section{Funktionelle und sozialmedizinische Bedeutung}

Die diabetische Retinopathie (DR) und Makulopathie, v. a. das diabetische Makulaödem (DME), stellen wie die Nephropathie oder Neuropathie mikrovaskuläre Spätkomplikationen des Diabetes mellitus (DM) dar. Sie treten in Abhängigkeit von Komorbidität und genetischem Hintergrund, wesentlich aber im Zusammenhang mit der Diabeteseinstellung bei jedem Betroffenen früher oder später auf. Wo und wie sich mikrovaskuläre diabetische Veränderungen primär nachweisen lassen, hängt besonders von Patientenalter und Diabetestyp ab [10].

Etwa 6\% der Weltbevölkerung leiden unter einem Diabetes mellitus, bis zu 97\% davon unter Typ 2. Im Laufe ihres Lebens erkranken mindestens $10 \%$ der Bevölkerung der westlichen Industriestaaten an einem DM [117]. Es muss davon ausgegangen werden, dass die Prävalenz des DM und damit die Bedeutung der Sekundärkomplikationen weltweit erheblich zunehmen werden. Nach Schätzungen der International Diabetes Foundation dürften bis zum Jahr 2025300 Mio Menschen betroffen sein [51]. Dies bedeutet weltweit eine Verdoppelung der Zahl im Vergleich zum Jahr 200o. Die größte Zunahme wird in den Ländern, die sich sozioökonomisch am schnellsten entwickeln [56], insbesondere in Osteuropa und Asien, aber auch in Afrika vermutet [74]; dies dürfte dort größte gesundheitspolitische Probleme mit sich bringen $[9,42,87,108,134]$. Dass diese Annahmen eher konservative Schätzungen sind, zeigt eine heute bereits übertroffene Modellrechnung aus dem Jahr
1997 für die Zahl der Diabetiker in den Jahren 2000 und 2010 auf vergleichbarer Grundlage (• Abb. 1, [9]). Wenn Mortalitätsrate und Diabetesinzidenz konstant bleiben, muss damit gerechnet werden, dass 38\% der heute 25-Jährigen im Laufe ihres Lebens einen DM entwickeln [86]. 20 Jahre nach Auftreten eines Diabetes (Typ 1 oder 2) ist eine Prävalenz der diabetischen Makulopathie von 5,9\% bei jüngeren und $7,5 \%$ bei älteren Patienten anzunehmen [61]. Bei Typ-1-Diabetes steigt die Prävalenz nach 25 Jahren auf 29\% für alle Makulödemformen und auf $17 \%$ für ein klinisch signifikantes Makulaödem (CSME; $[76,77]$ ). Dafür dürfte auch die heutige Versorgungskapazität der Ophthalmologie für Diabetiker nicht mehr ausreichend sein. Damit kommt dem Ver- ständnis der Diabeteskomplikationen und den Möglichkeiten zu ihrer Früherkennung und Behandlung zunehmende Bedeutung zu [64]. Die Entwicklung neuer Strategien zur Behandlung der mikro- und makrovaskulären Komplikationen ist entscheidend, um das Auftreten von Komplikationen hinauszuzögern und so dem Verlust der Arbeitsfähigkeit und damit in der überwiegenden Zahl der Fälle auch dem Verlust der sozialen und medizinischen Versorgung entgegenzuwirken. Entsprechend hat die vorliegende Übersicht zum Ziel, die funktionelle und sozialmedizinische Bedeutung der diabetischen Makulopathie und Retinopathie vorzustellen und neuere lokale und systemische Angriffspunkte für die Therapie und deren Effekt auf die Morbidität zu skizzieren.

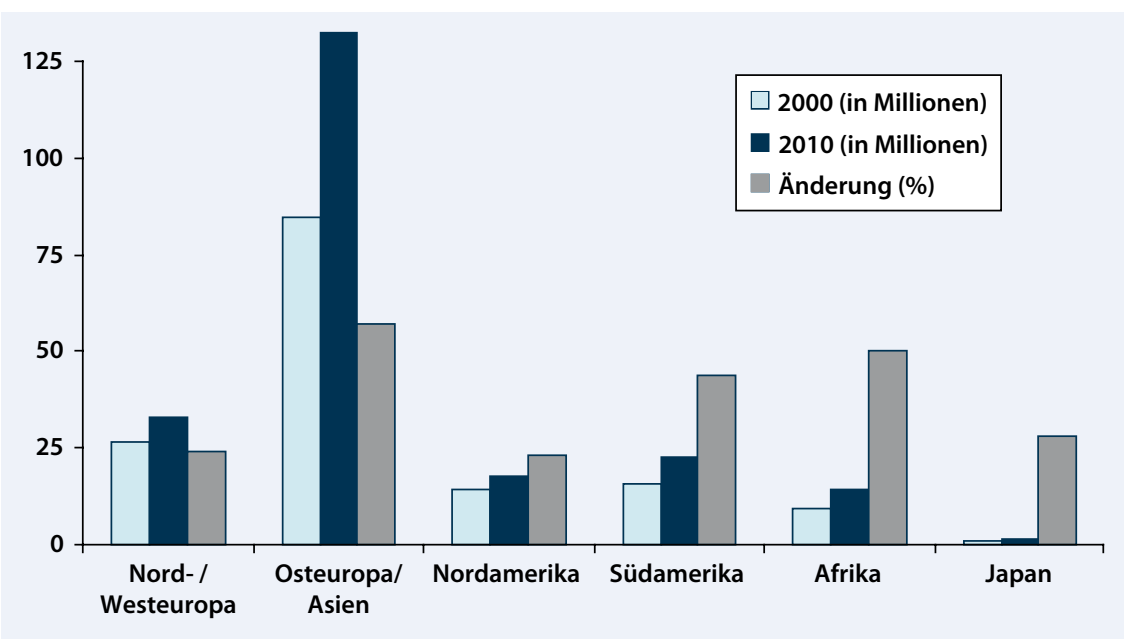

Abb. 1 A Die Projektion der Prävalenz des Diabetes mellitus für das Jahr 2010 hat vor 14 Jahren als eine eher konservative Schätzung die heutige Situation recht genau vorhergesagt. (Nach [9]) 
Ophthalmologe 2010 - 107:628-635 DOI 10.1007/s00347-010-2176-x

c) Springer-Verlag 2010

\section{J.G. Garweg · A. Wenzel \\ Diabetische Makulopathie und Retinopathie. Funktionelle und sozialmedizinische Bedeutung}

\section{Zusammenfassung}

Hintergrund. Die diabetische Makulopathie ist in den entwickelten Ländern führende Ursache für eine Erblindung vor dem Erreichen des Rentenalters. Die weltweit zunehmende Zahl von Diabetikern lässt vermuten, dass die Bedeutung der diabetischen Retinopathie und Makulopathie als Ursache einer schweren Sehbehinderung eher noch zunimmt.

Methode. Zu den Stichworten in der Kapitelüberschrift zu Epidemiologie, Bedeutung und Ursachen der Sehstörung bei diabetischer Makulopathie wurde eine Literatursuche durchgeführt, um Übersichtsartikel sowie kontrollierte Studien der letzten Jahre zusammenzustellen. Ziel war eine Übersicht über funktionelle und sozioökonomische Konsequenzen der diabetischen retinalen Mikroangiopathie und über neue therapeutische Strategien.
Ergebnisse. Erste Veränderungen in Richtung einer diabetischen Mikroangiopathie sind bereits früh nach Auftreten der Hyperglykämie nachweisbar. Langfristig verursacht sie schwere Organschäden. Für deren Behandlung werden wesentlich mehr Ressourcen benötigt als für die Behandlung der Hyperglykämie. Die diabetischen Mikroangiopathie verursacht darüber hinaus einen bemerkenswerten sozialmedizinischen Schaden. Der Früherkennung der vermehrten Gefäßpermeabilität bei Diabetikern kommt ein erheblicher Stellenwert für die Steuerung der Therapie zu. Für ein Hinauszögern der mikrovaskulären Komplikationen steht immer noch die Kontrolle der metabolischen Risikofaktoren Hyperglykämie und Hyperlipidämie sowie der Hypertonie im Vordergrund. Schlussfolgerungen. Moderne therapeutische Möglichkeiten erlauben heute eine medikamentöse Frühintervention mit dem Ziel, das Auftreten irreversibler mikrovaskulärer Schäden, namentlich der diabetischen Retinopathie und Makulopathie, hinauszuzögern. Das ophthalmologische Screening darf nicht mehr bei der Erkennung von Spätschäden diabetischer mikrovaskulärer Komplikationen am Auge beginnen. Eine enge interdisziplinäre Zusammenarbeit mit früher Implementation der neueren Therapieansätze bilden dabei die Grundlage für den therapeutischen Erfolg, bevor sich eine Sehbeeinträchtigung entwickelt.

\section{Schlüsselwörter}

Diabetische Mikroangiopathie · Diabetische Retinopathie · Diabetische Makulopathie . Intravitreale Therapie · Therapiekosten

\section{Diabetic maculopathy and retinopathy. Functional and sociomedical significance}

\section{Abstract}

Objective. The incidence of diabetic microvascular complications is expected to increase by $20-50 \%$ in the coming years. Diabetic macular edema (DME) is already a leading cause of blindness in the working-age population in developed countries, and its impact is expected to increase dramatically. Methods. Recent literature on the epidemiology and impact of diabetic microangiopathy (maculopathy) on visual function was reviewed to provide a comprehensive overview of the functional and socioeconomic consequences of diabetic retinal microangiopathy and new therapeutic strategies.

Results. The first changes indicating diabetic microangiopathy are detectable shortly after the development of hyperglycemia, and in the long term they induce severe organ damage. More resources are used for this condition's treatment than for the treatment of hyperglycemia, corresponding to an enormous sociomedical burden of disease. Early detection of increased retinal vascular permeability may help control treatment effects. The control of recognized risk factors for the development and progression of DME, namely hyperglycemia and hyperlipidemia, as well as of hypertension has remained the cornerstone of therapy and serves as the basis for preserving visual function.

Conclusions. Modern treatment options, begun early, may result in a remarkably delayed occurrence of irreversible diabetic microvascular pathologies, particularly diabetic retinopathy and maculopathy. Ophthalmological screening nowadays aims at earlier recognition of at-risk individuals to optimize the therapeutic strategy - that is, before visual impairment is imminent. Close interdisciplinary medical cooperation and implementation of new therapeutic options may provide the foundation for success in terms of maintaining visual function.

\section{Keywords}

Diabetic microangiopathy - Diabetic retinopathy - Diabetic maculopathy - Intravitreal therapy · Treatment costs 


\section{Steigende Inzidenz der diabetischen Retinopathie: Ursachen und Folgen}

Auch wenn die genauen Prävalenzannahmen zwischen einzelnen Publikationen schwanken [2], weisen sie den gemeinsamen geographischen Trend auf, dass in der weißen Bevölkerung von Europa und Nordamerika für das Jahr 2000 die niedrigste (4-6\%; Deutschland 5\%) und in $\mathrm{Pa}$ pua Neu Guiana (15,5\%), Mauritius (15\%), Bahrain (14,8\%), Mexico (14,2\%), Trinidad und Tobago $(14,1 \%)$ die höchste Diabetesprävalenz beobachtet wurde $[15,30$, 42, 68]. Während in Asien und Afrika eine Zunahme der Bevölkerungsdichte die Grundlage für die Annahme einer zunehmenden Inzidenz bildet, die v. a. die Altersgruppe der arbeitsfähigen 45- bis 65-Jährigen betreffen dürfte [19], wird eine steigende Inzidenz in Europa in den nächsten 25 Jahren trotz relativ stabiler Bevölkerungszahlen als Folge des zunehmenden Durchschnittsalters der Bevölkerung mit einem Inzidenzanstieg des Typ-2-Diabetes und seiner Komplikationen von mehr als $40 \%$ erwartet $[19,74]$. Wenn man davon ausgeht, dass die Inzidenz mikrovaskulärer Komplikationen unverändert bleibt, führt eine verbesserte Diabetestherapie zwangsläufig dazu, dass das Auftreten von Komplikationen länger überlebt wird. Damit muss in Zukunft sogar noch mit einem Anstieg des Anteils an Patienten mit ein oder mehreren diabetesassoziierten Komplikationen gerechnet werden [77, 111]. Eine Zunahme des Anteils an Diabetikern in der Bevölkerung wird in vergleichbarem Umfang auch für die USA prognostiziert. Auf der Basis der Zunahme der Prävalenzentwicklung zwischen 1980 und 1998 wird infolge der demographischen Veränderungen und der Zunahme der Bevölkerung erwartet, dass die Prävalenz um $65 \%$ von $4 \%$ im Jahr 2000 auf $7,2 \%$ im Jahr 2050 ansteigt [122]. Entsprechend vergleichbarer Annahmen der Bevölkerungsentwicklung, allerdings ohne erschwerende demographische Veränderungen, wurde für England ein Anstieg der Realkosten um 25\% und in Anbetracht der zurückgehenden Zahl Erwerbstätiger bis 2060 ein effektiver Kostenzuwachs von 40-50\% kalkuliert [16]. Als wesentliche Faktoren für die zunehmende Prävalenz in Europa und
Nordamerika werden das vielfach vorhandene Übergewicht insbesondere auch bei jüngeren und jugendlichen Typ-2-Diabetikern, aber auch eine schlechte Schulbildung und ein niedriger Sozialstatus verantwortlich gemacht [12, 21], welche das Komplikationsrisiko um das 1,5- bis 1,7fache erhöhen [82]. Deshalb wird in der Diabeteserziehung neben der Kontrolle von Blutzucker, Blutdruck und Serumlipiden zunehmend Wert auf eine intensive Lebensstilkontrolle gelegt [21]. In einer retrospektiven Querschnittuntersuchung aus Deutschland waren bei der Hälfte der untersuchten Diabetiker bereits schwere Komplikationen der Mikroangiopathie nachweisbar, die in o,8\% zu Herzinfarkt, in 1,3\% zu Apoplex und in o,8\% zu Amputationen geführt hatten. Bei $23 \%$ aller Patienten lagen mindestens zwei schwere Organmanifestationen vor [85].

Im Jahr 2000 wurden weltweit 2,9 Mio diabetesbedingte Todesfälle verzeichnet. Nach Angaben der WHO machen die diabetesassoziierten Todesfälle $5 \%$ aller Todesfälle, $2-3 \%$ in den ärmsten Ländern und $8 \%$ in USA und Kanada, aus. In der Altersgruppe der 35- bis 64-Jährigen steht Diabetes hinter 6-27\% aller Todesfälle [32]. Nach Untersuchungen aus Schweden und den USA wurde trotz einer mit 14-18\% stabilen Prävalenz kardiovaskulärer Erkrankungen ein Rückgang der Mortalität bei Typ-2-Diabetikern um bis zu $4 \%$ pro Jahr beobachtet $[112,113]$. Bei älteren Diabetikern ist das Vorhandensein eines diabetischen Makulaödems mit einer erhöhten Mortalität vergesellschaftet [61], was die allgemeine Bedeutung einer Mikroangiopathie für die Gesundheit von Diabetikern zeigt.

Eine Metaanalyse von 8 in den letzten 20 Jahren publizierten epidemiologischen Studien zur DR aus Europa und Nordamerika hat anhand der Auswertung von Fundusfotografien eine Prävalenz der DR von $28,7 \%$ ausgewiesen, wobei $9 \%$ der Betroffenen eine PDR und $17 \%$ ein DME aufwiesen. Deutlich niedrigere Prävalenzen fanden sich in Frankreich, was vermutlich an einem im Vergleich deutlich schlechteren Screening liegt. Die jährliche Inzidenz für das Neuauftreten einer DR bei Diabetikern liegt in Europa bei 2-6\% [31]. Nach kürzlich publizierten dänischen Daten liegt bei DM Typ 1 das kumulative Risiko einer nonproliferativen diabetischen Retinopa- thie (NPDR) nach 25 Jahren bei fast $46 \%$, das einer PDR bei 51,2\%, nur knapp 3\% weisen keine Zeichen einer DR auf [54]. Die Odds Ratio für die Entwicklung einer proliferativen diabetischen Retinopathie (PDR) liegt bei 2,1 pro erhöhtem Prozent HbA1c und bei 4,6 bei Vorliegen einer NPDR. Das Risiko einer Erblindung infolge Typ-1-Diabetes über 25 Jahre liegt bei 7,5\%. Berücksichtigt man auch die Todesfälle, liegt es sogar bei 9,5\% oder bei 4/100o Personenjahre. Dabei ist das Vorliegen eines DME oder einer DR mindestens so prädiktiv wie ein erhöhter HbA1c-Wert: Die Odds Ratio für eine Erblindung im Laufe von 25 Jahren lag bei 1,7 pro Prozent erhöhtem HbA1c, bei DME ohne PDR bei 6,2, bei zusätzlichem Vorliegen einer PDR bei 8,6. Im Vergleich dazu spielten Begleiterkrankungen, Dauer des Diabetes und Alter eine untergeordnete Rolle. Außerdem scheint eine diabetesbedingte Sehbehinderung auch mit einer erhöhten Mortalität vergesellschaftet zu sein [53]. Die kumulative Inzidenz einer Sehbehinderung liegt 25 Jahre nach Auftreten eines Typ-1-Diabetes bei 13\%, die einer schweren Sehbehinderung bei $3 \%$. Als Indikatoren der Progression wurden die Schwere der Retinopathie, die Präsenz einer Katarakt, der HbA1c-Anteil, Hypertonie und Rauchen, aber nicht eine Proteinurie identifiziert [77]. Es ist jedoch nicht nur die Katarakt mit dem Vorhandensein bzw. der Progression der DR assoziiert, zusätzlich verdoppelt möglicherweise die Kataraktoperation das Risiko einer Progression [66]. Auf der Basis nationaler Erhebungen in den USA aus dem Jahr 2005 und der Annahme einer steigenden Prävalenz der Grunderkrankung wird vom CDC eine Verdreifachung der Zahl von Patienten über 40 Jahre mit DR von 5 Mio im Jahr 2005 auf 16 Mio im Jahr 2050 erwartet. Die Zahl Sehbehinderter dürfte von 1,2 auf 3,4 Mio ansteigen. In der Altersgruppe der über 65-Jährigen ist sogar mit einer Vervierfachung zu rechnen [115]. Zusätzlich muss angenommen werden, dass der HbA1c-Grenzwert für die Entwicklung einer DR mit 5,5\% deutlich niedriger liegt als bisher angenommen wurde und klinisch realisierbar erscheint [22]. Nach Korrektur bezüglich der Diabetesdauer und des HbAic ist die Prävalenz von Sehbehinderungen in den letzten Jahren im Vergleich zu früher jedoch zurückgegangen, was als 
Zeichen einer besseren Therapie des Diabetes und seiner Begleiterkrankungen zu werten ist [78].

\section{Pathophysiologie der diabetischen Mikroangiopathie an der Netzhaut}

Die Manifestation der diabetischen Mikroangiopathie an den Augen ist in zivilisierten Ländern die häufigste Ursache für eine Sehbehinderung von Menschen unter 65 Jahren. Als Ursache findet sich ein DME als Ausdruck einer Gefäßschrankenstörung oder eine PDR als Folge ungezielten Gefäßwachstums (Angioneogenese). In der Folge der Gefäßveränderungen kommt es zu einem veränderten retinalen Blutfluss. Dieser geht mit Hypoxie im Gewebe einher, ist pathogenetisch noch nicht genau verstanden, spielt aber offensichtlich für die Progression der Retinopathie eine wichtige Rolle [29]. Offensichtlich führen erhöhte Blutglucosespiegel zu strukturellen und funktionellen Schäden an diabetischen Kapillaren. Die daraus resultierende Hypoxie im betroffenen Gewebe induziert die Freisetzung von VEGF (,vascular endothelial growth factor"), was die Gefäßpathologie verstärkt und die Gefäßproliferation fördert [26]. Der durch die Hyperglykämie induzierte Schaden ist die Folge eines Abbaus der überschüssigen Glucose über den Polyolstoffwechselweg, wodurch vermehrt sog. ,advanced glycation end products" (AGEs) und freie $\mathrm{O}_{2}$ Radikale anfallen. Parallel dazu kommt es zu einer Aktivierung der Proteinkinase C (PKC) und zu vermehrter Expression von Matrixproteinen, insbesondere Kollagen und Fibronectin, welche zur Verdickung der Basalmembran diabetischer Gefäße und damit Hypoxie im Gewebe beitragen (• Abb. 2, [58]). Zeitgleich kommt es zu einer vermehrten Expression vasoaktiver Mediatoren, v. a. Endothelin. Die vermehrte Freisetzung von VEGF reduziert die Stabilität der "tight junctions “ und führt damit zu einer gestörten Endothelbarriere. Der Austritt von Flüssigkeit ins Gewebe verstärkt die Hypoxie und die Extravasation von Blutbestandteilen unterstützt den entzündlichen Gewebsprozess. Basalmembranverdickung, vermehrte Gefäßpermeabilität und erhöhter Blutfluss wirken gemeinsam in Richtung eines zuneh-

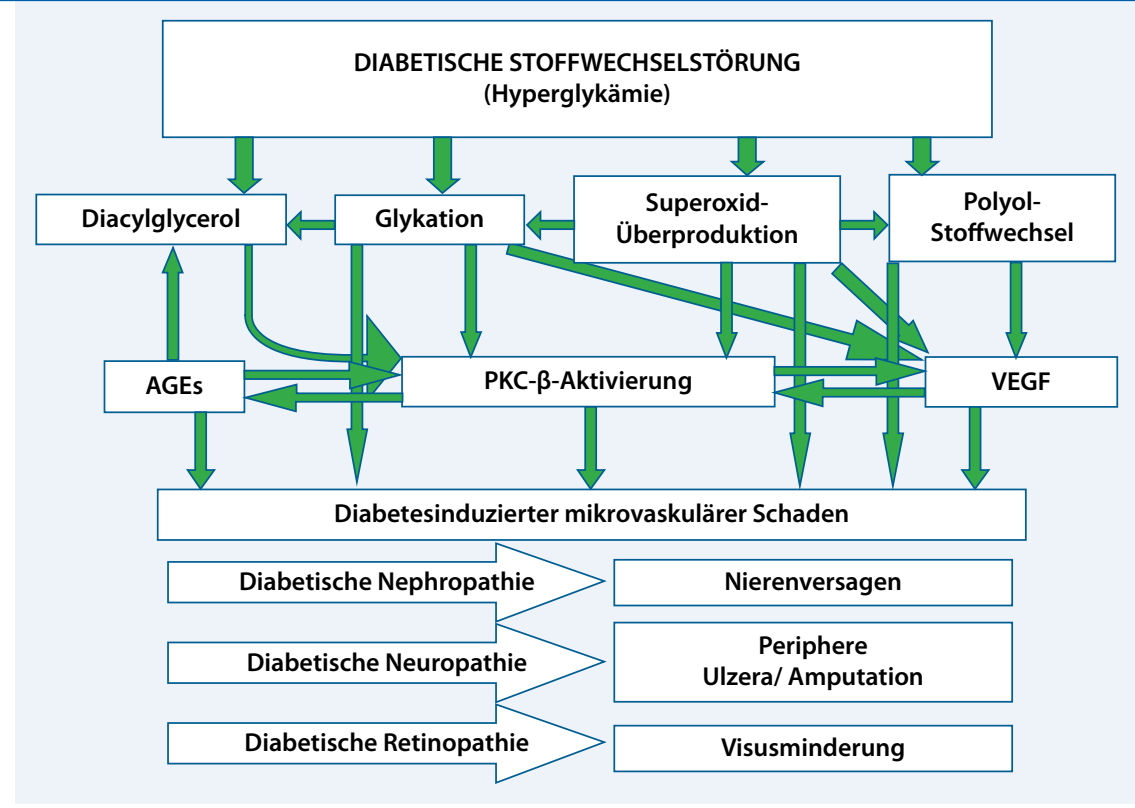

Abb. $2 \Delta$ Schema zur Pathophysiologie und den wichtigsten Konsequenzen der diabetischen Mikroangiopathie. PKC Proteinkinase C, AGEs „, advanced glycation end products", VEGF "vascular endothelial growth factor". (Nach [58])

mend gestörten Metabolismus im Gewebe [50]. Ursache und Bedeutung des entzündlichen Gewebsprozesses mit Hochregulierung multipler Entzündungsmarker im Zusammenhang mit der erhöhten Gefäßpermeabilität sind noch nicht geklärt. Die pharmakologische Beeinflussung erhöhter Konzentrationen von iNOS, COX2, ICAM-1, Caspase 1, NF-kB, Prostaglandin E2, IL-1 $\beta$ und erhöhter Zytokinspiegel sowie der vermehrten Leukostase in diabetischen Gefäßen bietet aber klare Evidenz für ihre pathogenetische Bedeutung in den Frühstadien der DRP, da mit der Behandlung die Entstehung der Gefäßveränderungen im Tiermodell verhindert werden kann. Deren klinisches Potenzial ist derzeit unklar $[1,71]$.

Eine Anreicherung von AGEs ist sehr früh in der Entstehung der diabetischen Retinopathie und Makulopathie zu finden und bereits wenige Monate nach Auftreten der Hyperglykämie vorhanden. Sie führt unter anderem zu einer Verdichtung der vitreoretinalen Grenzfläche und damit vermutlich zu gestörter Sauerstoffdiffusion, die bereits früh einen subklinischen neurovaskulären Schaden verursacht [29]. Ein Anstieg der intrazellulären Glucosespiegel führt außerdem zur Bildung freier Sauerstoffradikale und der Entstehung von oxidativem Stress und aktiviert die PKC $[36,98,107,109,135]$. Die Folge ist eine Erhöhung der Konzentration von VEGF, ei- ne endotheliale Funktionsstörung, vermehrte Leukozytenadhäsion, erniedrigte Konzentration von „pigment epithelium-derived factor" (PEDF) und eine erhöhte Aktivität der PKC. Folge der diabetesbedingten Veränderungen der Gefäßwandstruktur und -funktion ist die oben erwähnte vermehrte Gefäßpermeabilität und damit der Zusammenbruch der BlutRetina-Schranke (BRS). Eine neuronale Dysfunktion der Netzhaut ist allerdings bereits vor Auftreten erster klinischer Gefäßveränderungen nachweisbar [46]. Andere Zeichen einer gestörten neuronalen Funktion, unter anderem eine verlängerte Latenz der B-Welle [13] und gestörtes Farb- und Kontrastsehen [65, 114, 124], werden ebenfalls bereits in frühen Diabetesstadien beobacht, sodass heute neben bzw. bereits vor der vaskulären auch eine neuronale bzw. neurodegenerative Komponente angenommen wird [46, 139]. Ein Fortschreiten der Makulopathie und Retinopathie muss erwartet werden, wenn infolge der oben genannten Veränderungen Hypoxie, gestörter Blutfluss, retinale Ischämie und Entzündung zunehmen. In Tiermodellen konnte gezeigt werden, dass AGEs die Aktivierung sämtlicher oben erwähnter Prozesse fördern. Deren Rezeptoren, die sog. RAGE, lösen in der Folge auch entzündliche Gewebsreaktionen aus. Dies erklärt die Verbindung zwischen Diabetes und entzündlicher Gewebsreaktion 


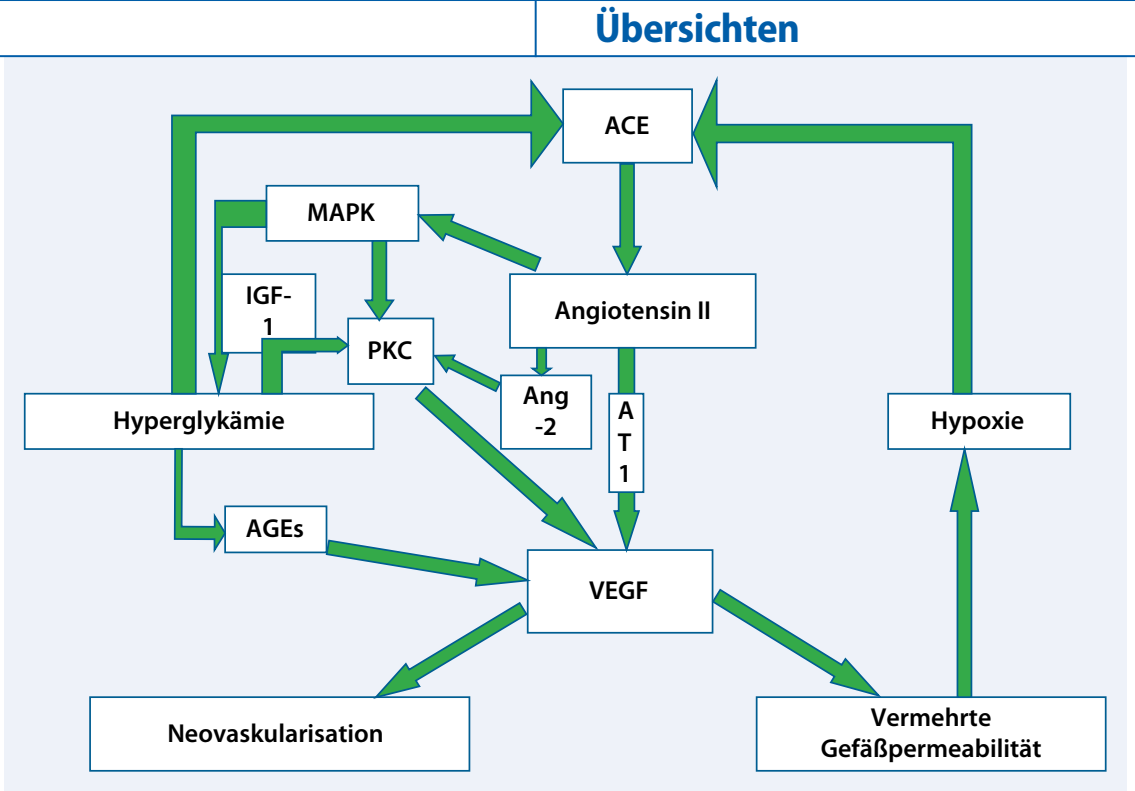

Abb. $3 \Delta$ Schema der systemischen Angriffspunkte zur Prophylaxe und Behandlung der diabetischen Mikroangiopathie. $A C E_{\text {" }}$ angiotensin converting enzyme", $M A P K_{\text {"I }}$ mitogen-activated protein kinase, PKC Proteinkinase C, Ang-2 Angiopoietin-2, AGEs ", advanced glycation end products, VEGF "vascular endothelial growth factor", AT1 Angiotensin II Typ 1

und bietet neue Möglichkeiten für zusätzliche therapeutische Ansätze [73, 93, 109, $110,139,140]$.

Als histologisches Korrelat der klinisch sichtbaren Veränderungen finden sich die oben bereits erwähnte Basalmembranverdickung retinaler Blutgefäße [43], eine $\mathrm{Ab}$ nahme der Perizytenanzahl im Vergleich zu den Endothelzellen und in der Folge Mikroaneurysmen als erste sichtbare Zeichen der Mikroangiopathie [59]. Vor ihrer Entstehung lässt sich in der Netzhaut ein erhöhter VEGF-Spiegel nachweisen [8].

Da die hier für die Netzhaut beschriebenen pathobiochemischen und pathophysiologischen Prozesse unspezifisch Schaden an allen kleineren und größeren Gefäßen des Körpers verursachen, überrascht es nicht, dass das Vorhandensein eines DME und einer DRP mit verschiedenen anderen vaskulären Diabeteskomplikationen assoziiert ist (• Abb. 2). Das DME ist z. B. mit einem erhöhten Risiko ischämischer kardiovaskulärer Ereignisse vergesellschaftet [61]. Andererseits haben pathophysiologisch basierte systemische Therapieansätze auch zu einer Stabilisierung der Erkrankung an der Netzhaut geführt, was deren pathogenetische Rolle bestätigt: Zellkultur- und tierexperimentelle Studien haben gezeigt, dass der Lipidstoffwechsel mit der Hochregulierung der Entzündungsmarker zusammenhängt [83]. Parallel dazu wurde klinisch die Wirkung der Statine auf den Verlauf der diabetischen Mikro- angiopathie im Sinne einer Reduktion von kardiovaskulärer Morbidität und Mortalität nachgewiesen [25]. Eine lipidsenkende (Fenofibrat)-Therapie senkt die Erfordernis von Laserinterventionen von 4,9 auf 3,4\% (Hazard Ratio o,69) und das Risiko einer DME-Progression unabhängig von den Serumlipidspiegeln [70].

\section{Begleiterkrankungen und beeinflussbare Risikofaktoren}

An dem Auftreten einer diabetischen Stoffwechselstörung ist nichts zu ändern, aber die Geschwindigkeit der Progression einer diabetischen Mikroangiopathie lässt sich durch eine konsequente Behandlung der Blutzuckereinstellung und der Kofaktoren beeinflussen (- Abb. 3). Dies hat eine Metaanalyse von 28 Studien mit 27.00o Patienten gezeigt [36]. Dabei ist als Ziel der Therapie eine straffe Blutzucker-, Blutdruck- und Serumlipideinstellung auf (fast) normale Werte sowie eine Änderung der Lebensgewohnheiten mit Kontrolle der Diät und Minimierung toxischer Einflüsse wie Rauchen und Alkohol. Der Erfolg einer strengen Diabetestherapie hinsichtlich Morbidität und Mortalität rechtfertigt die dadurch verursachten Aufwände, insbesondere bezüglich kardiovaskulärer Begleiterkrankungen, Hypertonie und Hypercholesterinämie $[55,136]$. Eine straffe Blutzuckereinstellung reduziert das Risiko für kardivaskuläre Ereignisse um 42\%, für Herzinfarkt und Apoplex sogar um $57 \%$ [95]. Das relative Risiko für mikrovaskuläre Schäden an den Nieren ließ sich damit über 7,8 Jahre um 61\%, das einer Retinopathie um $58 \%$ und das einer Neuropathie um 63\% reduzieren [129]. Umgekehrt ist eine DR mit einer erhöhten kardiovaskulären Morbidität vergesellschaftet [49]. Durch die verbesserten therapeutischen Optionen ist zu erklären, dass Inzidenz und Progression von PDR und schwerem Sehverlust im Lauf der letzten 35 Jahre zurückgingen [136]. Hypertonie, kardiovaskuläre Erkrankungen, die Dauer des Diabetes und die Höhe des glykolysierten Hämoglobins (HbA1c) sind eng mit der Schwere und Progression der DR korreliert. Dies zeigt, dass Makro- und Mikroangiopathie sich gegenseitig verstärkende Multiorganmanifestationen des Diabetes in Abhängigkeit der Funktion von Nieren, Herz und Augen sind [4, 23, 76]. Daraus lässt sich ableiten, dass, je besser das Verständnis pathophysiologischer Mechanismen und je besser die Therapie der Grunderkrankung und der Begleiterkrankungen ist, sich desto mehr langfristig die Progression der DR verlangsamen lässt [23]. So sinkt das Risiko einer DR mit einer Verbesserung der Blutzucker- und Blutdruckkontrolle im Laufe der Erkrankung [14, 75]. Im klinischen Alltag wird allerdings die Patienten-Compliance - gemessen anhand der Blutzuckereinstellung (HbA1c-Werte) selbst nach dem Auftreten schwerer vasookklusiver Komplikationen des Diabetes nicht besser [69]. So erklärt sich, dass das Vorhandensein einer PDR und Proteinurie mindestens bei Typ-1-Diabetes die Lebenserwartung verkürzt, wohingegen das Vorliegen einer NPDR die Lebenserwartung über 25 Jahre offensichtlich nicht beeinflusst [52].

Das Risiko von Diabeteskomplikationen korreliert unabhängig, aber additiv mit der Blutzucker- und Blutdruckeinstellung [3, 14], wobei für Entwicklung und Progression der diabetischen Retinopathie und Makulopathie insbesondere die Hypertonie ein wesentlicher Risikofaktor ist $[90,91]$. Deshalb werden heute als Obergrenze einer straffen Blutdruckeinstellung bei Diabetes Blutdruckwerte von 130/80 mmHg angenommen [28, 94, 127]. In der Praxis ist dies allerdings nicht immer einfach, wenn man berücksichtigt, dass 
Body-Mass-Index (BMI, korreliert mit dem Körpergewicht) und Gefäßvorschädigung (Vorhandensein einer Mikroalbuminurie) eine entscheidende Rolle für die Blutdruckeinstellung spielen [97]. Hintergrund hierfür ist, dass die Aktivierung von Angiotensin-II-Typ-1-Rezeptoren (AT1-R) in retinalen Endothelzellen und Perizyten in Zusammenhang mit dem Auftreten mikrovaskulärer Komplikationen gebracht wird [24]. In tierexperimentellen Studien konnte gezeigt werden, dass eine Blockade des Renin-Angiotensin-Systems [mit Angiotensin-converting-enzyme- (ACE-)Inhibitoren oder AT1-Rezeptor-Blockern) zu verbesserter Hämodynamik, verminderter Gefäßpermeabilitätund reduzierter Leukostase führt $[72,105,144]$. Eine Therapie mit ACE-Hemmern verzögert zwar nicht das Auftreten einer Nephropathie, aber die Progression der diabetischen Retinopathie [91, 116, 144]. AT1-Rezeptor-Antagonisten können das Neuauftreten einer DRP verzögern; auf der Basis der experimentellen Ergebnisse kann jedoch nicht gezeigt werden, dass sie die Progression einer DRP über 5 Jahre aufhalten [20, 121, 137]. Da seit einigen Jahren klinisch ein positiver Effekt der Statine auf das Auftreten vaskulärer Komplikationen bekannt ist, wurden diese heute recht breit als Lipidsenker verwandten 3-Hydroxy-3-Methylglutaryl-Coenzym-A- (HMG-CoA-)Reduktase-Inhibitoren auch tierexperimentell untersucht. Danach scheinen sie die Blut-Retina-Barriere bei DRP unabhängig vom Vorhandensein einer Fettstoffwechselstörung über eine noch unklare, möglicherweise antientzündliche Wirkung zu stabilisieren [7, 127] und das Risiko kardiovaskulärer Komplikationen erheblich zu reduzieren [79]. Eine Reduktion der diabetischen Gefäßwandleckage wird auch durch Ruboxistaurin, einen selektiven Proteinkinase-C- $\beta$-Inhibitor, erreicht, wodurch auch das Risiko eines Visusverlusts bei Patienten mit mäßiger bis schwerer nichtproliferativer diabetischer Retinopathie reduziert wird [99, 106, 107].

In der Praxis besteht ein erhebliches Problem für die strenge Kontrolle des Diabetes sowie der vaskulären Risikofaktoren (Hypertonie und Hyperlipidämie) darin, dass eine Näherung an die Zielwerte für mehr als die Hälfte der Betroffenen eine erhebliche Medikamentenbelastung mit mehr als 5 Medikamenten täglich bedeu- tet. Die Obergrenze der Blutfettwerte wird eher als die des Blutzuckers und deutlich besser als die des Blutdrucks erreicht [128]. Viele Patienten erreichen diese Vorgaben nicht wegen einer entsprechend der Leitlinien nicht ausreichend aggressiven Behandlung insbesondere der Begleiterkrankungen durch ihre behandelnden Ärzte [102]. Ein weiteres Problem bei der komplexen Therapie des Diabetes und seiner Begleiterkrankungen sind die Nebenwirkungen der Therapie, wie am Beispiel des Glitazon kürzlich gezeigt wurde. Unter dieser Therapie ist das Risiko eines diabetisches Makulaödems, unabhängig von der Blutzuckerkontrolle, möglicherweise doppelt so hoch wie unter anderen oralen Antidiabetika [41].

\section{Neuere lokale therapeutische Ansätze}

Goldstandard in der Therapie diabetischer Netzhautveränderungen ist seit dem Nachweis der Effizienz in der ETDRS-Studie immer noch die Lasertherapie [34]. Bei vorwiegend ischämischer Makulopathie ist eine Laserkoagulation im Bereich der Makula nicht indiziert. Ansonsten stellt die Lasertherapie bisher die einzige etablierte Therapie bei klinisch signifikantem Makulaödem (CSME) dar, obwohl sie insbesondere bei diffusem Makulaödem mittelbis langfristig keine Visusverbesserung bewirkt [92]. Dabei hat ein Auge mit schlechtem Visus eine gute Aussicht auf Funktionsverbesserung, wohingegen guter Visus vor der Koagulation und ausgeprägtes Makulaödem auf eine schlechte funktionelle Prognose hinweisen [5].

Die Laserwirkung führt man zurück auf eine Stimulation der Endothelzellreplikation bei direkter Koagulation von Aneurysmen, die Anregung der Phagozytoseaktivität im retinalen Pigmentepithel, eine Verbesserung der Sauerstoffversorgung in der inneren Retina durch Zerstörung des Pigmentepithels und der Rezeptoren und vermutlich v. a. auf die Reduktion angiogenetischer Faktoren durch Zerstörung der hypoxischen Netzhaut [104, 125, 126]. Dabei scheint eine besonders schonende Argonlasertechnik (ALK) auch einen geringeren Effekt auf das Makulaödem zu haben, was sich über 12 Monate im Visus allerdings nicht niederschlägt [138]. Die der- zeit vermehrt diskutierte SubthresholdMikropuls-Diodenlaser-Therapie hat bezüglich der Langzeitergebnisse gegenüber konventioneller Lasertherapie weniger Narbenbildung, ansonsten aber bisher keine klare Überlegenheit gezeigt [38, 39, 101, 120]. Auf eine fokale ALK reagiert ein $\mathrm{Ma}-$ kulaödem über einen Zeitraum von $6 \mathrm{Mo-}$ naten weniger gut als auf eine intravitreale Triamcinolon-Injektion (IVTA) oder eine Vitrektomie, ohne dass sich dies im Visus bemerkbar macht; das Risiko einer Ödemzunahme ist aber kleiner als nach Vitrektomie [18]. Über einen Zeitraum von 2 Jahren zeigt sich allerdings ein Vorteil der ALK gegenüber der IVTA in Bezug auf den Visus [33]. Im Vergleich zu einer alleinigen panretinalen ALK scheint die Kombination von panretinaler ALK und intravitrealer Triamconolon-Injektion mindestens über einen Zeitraum von 9 Monaten funktionell vorteilhaft zu sein [57, 88, 143]. Die kurzfristigen Vorteile der IVTA gehen mit einer Reihe ernstzunehmender auch langfristig relevanter Nebenwirkungen einher [142], weshalb die TriamcinolonMonotherapie heute als Second-Line-Therapie bei Versagen anderer Optionen gesehen wird $[47,48]$. Der Stellenwert der intravitrealen Triamcinolon-Acetonid-Injektion im Vergleich zur Lasertherapie bei DME ist unklar; vermutlich ist die Kombination beider Therapien effektiver als jede einzeln angewandt $[17,34]$.

Da die fokale ALK als alleinige Lokaltherapie in vielen Fällen zwar die Funktion stabilisieren, aber nicht bessern kann und die IVTA wegen ihrer Nebenwirkungen kein geeignetes Mittel zu einer dauerhaften Stabilisation der Retinopathie ist, ist der Bedarf nach alternativen und ergänzenden lokalen Therapien von zunehmender Bedeutung [119]. Dafür gibt es verschiedene Angriffspunkte [6,37]. Derzeit stehen intravitreale Steroidinjektionen, Pars-plana-Vitrektomie, RNA-basierte Therapien, orale Proteinkinase-CInhibition, Hydroxy-3-Methyl-GlutarylCoenzym-A-Reduktase-Inhibitoren und nichthormonelle antiinflammatorische Therapien (Anti-IL-2, Anti-IL-6) und in den letzten Jahren v. a. Anti-VEGF-Injektionen unter Evaluation [37].

Die intravitreale Injektion von Antikörpern gegen VEGF [Bevacizumab (Avastin ${ }^{\oplus}$ ), Ranibizumab (Lucentis $\left.\left.{ }^{\circledast}\right)\right]$ oder Ap- 
tameren [Pegaptanib (Macugen $\left.{ }^{\circ}\right)$ führt zu einem Rückgang der Netzhautdicke und Visusanstieg innerhalb der ersten 3 Monate. Danach ist aber vermutlich ein schrittweiser Wirkungsrückgang zu erwarten, wenn nicht konsequent die erhöhten okulären VEGF-Spiegel unterdrückt, d. h. häufige Injektionen vorgenommen werden $[11,27,96]$. Entsprechend ist anfänglich Triamcinolon der Anti-VEGFTherapie hinsichtlich der Anzahl an Injektionen bezüglich der Reduktion der Netzhautdicke und der Visus-Erholung pro Behandlung überlegen $[35,103]$. Offensichtlich übersteigt aber unter monatlichen Lucentis-Injektionen der Visusgewinn von 10,3 ETDRS-Buchstaben über 12 Monate den Effekt jeglicher bisheriger Therapien (auch Triamcinolon) bei weitem [89]. Gemäß den Sechsmonatsergebnissen der READ-2-Studie scheint die zweimonatliche intravitreale Anti-VEGFInjektion (Lucentis ${ }^{\circ}$ ) der Kombination von Laser und Anti-VEGF überlegen zu sein [96]. Auf die noch nicht publizierten Ergebnisse der RESTORE-Studie, die in 3 Armen Laser-, Lucentis-Mono- und Kombinationstherapie vergleicht, darf man gespannt sein. Der Effekt einer Bevacizumab-Therapie scheint dem der Ranibizumab-Therapie vergleichbar zu sein [123]; für einen abschließenden Vergleich liegen aber zu wenig Daten vor. Wenn bereits eine Vitrektomie mit ILM-Peeling zur Behandlung eines CSME durchgeführt wurde, zeigt eine VEGF-Inhibition (Avastin) auf die Reduktion der Netzhautdicke keinen Effekt mehr [141]. Nach den verfügbaren kontrollierten Studien wird die beste Langzeitstabilisierung bei DME jedoch nicht durch eine dieser Maßnahmen allein, sondern durch die Kombination einer optimalen Kontrolle der Komorbidität, der neuen pharmakologischen intravitrealen Therapieoptionen und der Lasertherapie erreicht $[44,100]$.

\section{Gesundheitskosten der diabetischen Mikroangiopathie}

Die sozialmedizinischen Folgen dieser multifaktoriellen Systemerkrankung werden oft bei weitem unterschätzt [84]: Die diabetische Retinopathie ist weltweit die fünfthäufigste Erblindungsursache. Die diabetische Nephropathie ist die Hauptur- sache der chronischen Niereninsuffizienz. Periphere Ulzera an den unteren Extremitäten infolge einer diabetischen Neuropathie sind die häufigste Indikation für eine Amputation [23, 58].

Mit den heutigen Therapiemöglichkeiten können diabetische Retinopathie, Nephropathie und Neuropathie nicht aufgehalten werden. Die neuen therapeutischen Optionen der letzten 15 Jahre bieten in Kombination mit einer ausgebauten Standardtherapie des Diabetes und seiner Komplikationen für die $\mathrm{Zu}$ kunft jedoch berechtigte Hoffnung, eine Progression der diabetischen Mikroangiopathie deutlich zu verlangsamen und damit die Sehfunktion und Lebensqualität zu stabilisieren und die Lebenserwartung deutlich zu verbessern $[63,127]$. Die Therapie der diabetischen Dyslipidämie mit hohen Triglyzerid- und erniedrigten LDL-Cholesterol-Spiegeln hat einen positiven Effekt auf die Mikroangiopathie. Die positive Wirkung einer FenofibratTherapie auf die Makulopathie und Retinopathie und ein Effekt der Statine und der Fibrate auf die diabetische Nephropathie sind etabliert [40]. Eine intensivierte Therapie mit straffer Kontrolle der Risikofaktoren ist entsprechend kosteneffektiver als die konventionelle Therapie [45]. Die Kosteneffektivität des Effekts dieser Zusatztherapien auf die Mikro- und Makroangiopathie ist offensichtlich, auch wenn für die Therapie des Diabetes ohne Berücksichtigung der diabetesassoziierten Begleiterkrankungen in den USA jährlich 90 und für die kardiovaskulärer Erkrankungen 250 Mrd Dollar ausgegeben werden. Die Kosteneffektivität wird insbesondere bei einer präventiven Therapiestrategie offensichtlich, noch bevor die Angiopathie sich manifestiert [62]. Trotz aller therapeutischer Optionen bleibt auf die Dauer das Risiko mikrovaskulärer Erkrankungen erheblich. Insbesondere fehlen noch Möglichkeiten zur Beeinflussung der peripheren Neuropathie. Dies liegt vermutlich daran, dass die pathophysiologischen Zusammenhänge für das Auftreten und Fortschreiten der diabetischen Mikroangiopathie auch heute noch weitgehend ungeklärt sind [40]. Die Gesundheitskosten von Patienten mit diabetischen mikrovaskulären Komplikationen liegen in Deutschland um das 3- bis 4-fache (bis zu 78\%) über denen von Diabetespatienten ohne Angiopathie [130, 131]. Für die nächsten 25 Jahre muss einerseits infolge der sich ändernden Altersstruktur mit einer Verdoppelung der Zahl an Diabetikern in der Bevölkerung und andererseits infolge der neuen diagnostischen und therapeutischen Möglichkeiten in den westlichen Ländern mit einer Verdreifachung der diabetesassoziierten Kosten gerechnet werden. In den USA wurden 2009113 Mrd Dollar für die Behandlung diabetesbezogener Erkrankungen ausgegeben, im Jahr 2034 dürften es bereits 336 Mrd Dollar werden [67]. Berücksichtigt man die zusätzlich vorhandenen indirekten Krankheitskosten (Arbeitsausfall, gesundheitliche Einschränkung im Alltag und permanente Behinderung, Mortalität), steigen die Kosten um weitere $35 \%$. Im Jahr 2002 lagen die Kosten für die Behandlung des Diabetes in den USA mit insgesamt 13.243 US \$ pro Person um fast 10.000 \$ über denen für andere Erkrankungen und damit um das 2,4-fache über den Gesundheitskosten der gleichen Altersgruppe ohne Diabetes [64].

Ähnliche Zahlen werden auch für Deutschland genannt: Für das Jahr 2001 wurden direkte Kosten von 5262 EUR und indirekte diabetesassoziierte Kosten von 5019 EUR ermittelt. Damit liegen sie auch in Deutschland um das 2- bis 4fache über den Kosten von Patienten der gleichen Altersgruppe ohne Diabetes, was $200114,2 \%$ der gesamten Gesundheitskosten entsprach $[81,84]$. Dabei muss von einer Kostensteigerung von $1 \%$ pro Jahr ausgegangen werden [84]. Die Glucoseselbstmessung trägt trotz vermehrter Diagnostikkosten erheblich zu einer Therapiekosteneinsparung von bis $\mathrm{zu} 25 \%$ bei [132, 133]. In der CoDiM-Studie kostete die direkte Behandlung der Hyperglykämie 469 EUR; für die Behandlung von Diabeteskomplikationen wurden darüber hinaus im Mittel 3730 EUR ausgegeben, wobei je nach Komplikation bei jedem 20. Patienten Kosten von über 20.00o EUR entstehen [80].

Die Behandlung des DME verursacht im ersten Jahr zusätzliche Kosten von 31\%, innerhalb 3 Jahren immer noch von $29 \%$ nicht zuletzt durch zusätzlich erforderliche Diagnostik und Therapieänderungen [6o]. Zwischen 2000 und 2004 nahm die 
Zahl der Patienten, die wegen eines DME eine intravitreale Therapie erhielten, von $1 \%$ auf $13 \%$ zu. Die Anwendung der optischen Kohärenztomographie (OCT) stieg von $2,5 \%$ auf über $40 \%$, wohingegen die Häufigkeit einer Laserbehandlung in dieser Zeit eher abnahm [118].

\section{Fazit für die Praxis}

Die Behandlung des Diabetes und der diabetischen Retinopathie ist nach wie vor unbefriedigend. 50 Jahre nach ihrer Einführung stellt die Ablation kranker Netzhaut durch Laserkoagulation den Kernpunkt der Therapie der proliferativen Retinopathie dar. Immerhin gibt es zahlreiche neuere Therapien der metabolischen Grunderkrankung, die den Verlauf verzögern können. Mit neuen lokalen Maßnahmen lässt sich eine brauchbare Funktion mindestens über mehrere Jahre nach Auftreten eines DME wiederherstellen. Vermutlich wird ein weiterer Quantensprung in der Therapie der Retinopathie erst durch einen tieferen Einblick in die Pathophysiologie, insbesondere in die Frühveränderungen der DRP, möglich. Offensichtlich prädisponieren die spezifische Anatomie und Physiologie der Netzhaut zu einer besonderen Vulnerabilität durch die Folgen der Stoffwechselstörung. Betrachtet man die sozialmedizinische Bedeutung der Erkrankung, sind aber sicherlich alle Bemühungen um neue Therapien gerechtfertigt. Basis aller klinischen Bemühungen muss jedoch eine strenge Kontrolle des Diabetes und seiner Begleiterkrankungen sein, die allein über einen Zeitraum von nur 5 Jahren bereits eine Reduktion diabetesassoziierter Komplikationen von $10 \%$ bewirkt $[3,14]$.

\section{Korrespondenzadresse \\ Prof. Dr. J.G. Garweg}

Swiss Eye Institute, Berner Augenklinik am Lindenhofspital, Universität Bern Bremgartenstraße 119, 3012 Bern Schweiz garweg@augenklinik-bern.ch

Interessenkonflikt. Der korrespondierende Autor gibt an, dass kein Interessenkonflikt besteht.

\section{Literatur}

1. Adamis AP, Berman AJ (2008) Immunological mechanisms in the pathogenesis of diabetic retinopathy. Semin Immunopathol 30:65-84

3. ADVANCE Collaborative Group, Patel A, MacMahon $S$ et al (2008) Intensive blood glucose control and vascular outcomes in patients with type 2 diabetes. N Engl J Med 358:2560-2572

5. Aiello LP, Edwards AR, Beck RW et al (2010) Factors associated with improvement and worsening of visual acuity 2 years after focal/grid photocoagulation for diabetic macular edema. Ophthalmology [Epub ahead of print]

14. Beulens JW, Patel A, Vingerling JR et al (2009) Effects of blood pressure lowering and intensive glucose control on the incidence and progression of retinopathy in patients with type 2 diabetes mellitus: a randomised controlled trial. Diabetologia 52:2027-2036

16. Boyle JP, Honeycutt AA, Narayan KM et al (2001) Projection of diabetes burden through 2050: impact of changing demography and disease prevalence in the U.S. Diabetes Care 24:1936-1940

18. Browning DJ, Fraser CM, Powers ME (2006) Comparison of the magnitude and time course of macular thinning induced by different interventions for diabetic macular edema: implications for sequence of application. Ophthalmology 113:1713-1719

20. Chaturvedi N, Porta M, Klein R et al (2008) Effect of candesartan on prevention (DIRECT-Prevent 1) and progression (DIRECT-Protect 1) of retinopathy in type 1 diabetes: randomised, placebocontrolled trials. Lancet 372:1394-1402

22. Cheng YJ, Gregg EW, Geiss LS et al (2009) Association of $\mathrm{A} 1 \mathrm{C}$ and fasting plasma glucose levels with diabetic retinopathy prevalence in the U.S. population: Implications for diabetes diagnostic thresholds. Diabetes Care 32:2027-2032

23. Cheung N, Wong TY (2008) Diabetic retinopathy and systemic vascular complications. Prog Retin Eye Res 27:161-176

24. Clermont A, Bursell SE, Feener EP (2006) Role of the angiotensin II type 1 receptor in the pathogenesis of diabetic retinopathy: effects of blood pressure control and beyond. J Hypertens Suppl 24:S73-S80

28. Currie CJ, Peters JR, Tynan A et al (2010) Survival as a function of $\mathrm{HbA}(1 \mathrm{c})$ in people with type 2 diabetes: a retrospective cohort study. Lancet 375:481-489

29. Curtis TM, Gardiner TA, Stitt AW (2009) Microvascular lesions of diabetic retinopathy: clues towards understanding pathogenesis? Eye (Lond) 23:1496-1508

34. Diabetic Retinopathy Clinical Research Network (DRCR.net), Beck RW, Edwards AR et al (2009) Three-year follow-up of a randomized trial comparing focal/grid photocoagulation and intravitreal triamcinolone for diabetic macular edema. Arch Ophthalmol 127:245-251

37. El-Asrar AM, Al-Mezaine HS, Ola MS (2009) Changing paradigms in the treatment of diabetic retinopathy. Curr Opin Ophthalmol 20:532-538

45. Gaede P, Valentine WJ, Palmer AJ et al (2008) Cost-effectiveness of intensified versus conventional multifactorial intervention in type 2 diabetes: results and projections from the Steno- 2 study. Diabetes Care 31:1510-1515
47. Gillies MC, Simpson JM, Gaston C et al (2009) Five-year results of a randomized trial with open-label extension of triamcinolone acetonide for refractory diabetic macular edema. Ophthalmology 116:2182-2187

53. Grauslund J, Green A, Sjølie AK (2009) Blindness in a 25-year follow-up of a population-based cohort of Danish type 1 diabetic patients. Ophthalmology 116:2170-2174

57. Grover D, Li T, Chong C (2008) Intravitreal steroids for macular edema in diabetes. Cochrane Database Syst Rev (1):CD005656

59. Hammes HP (2005) Pericytes and the pathogenesis of diabetic retinopathy. Horm Metab Res 37(Suppl 1):39-43

60. Hauner H (2006) Die Kosten des Diabetes und seiner Komplikationen in Deutschland. DMW 131:240-242

61. Hirai FE, Knudtson MD, Klein BE, Klein R (2008) Clinically significant macular edema and survival in type 1 and type 2 diabetes. Am J Ophthalmol 145:700-706

66. Hong T, Mitchell P, Loryn T de et al (2009) Development and progression of diabetic retinopathy 12 months after phacoemulsification cataract surgery. Ophthalmology 116:1510-1514

69. Jørgensen TM, Grauslund J, Sjølie AK et al (2009) Major diabetes-related vascular events do not improve glycaemic control in a population-based cohort of type 1 diabetic individuals. Scand J Clin Lab Invest 1-4 [Epub ahead of print]

78. Klein R, Lee KE, Knudtson MD et al (2009) Changes in visual impairment prevalence by period of diagnosis of diabetes: the wisconsin epidemiologic study of diabetic retinopathy. Ophthalmology 116:1937-1942

86. Magliano DJ, Shaw JE, Shortreed SM et al (2008) Lifetime risk and projected population prevalence of diabetes. Diabetologia 51:2179-2186

90. Matthews DR, Stratton IM, Aldington SJ et al (2004) Risks of progression of retinopathy and vision loss related to tight blood pressure control in type 2 diabetes mellitus: UKPDS 69. Arch Ophthalmol 122:1631-1640

102. Ott P, Benke I, Stelzer J et al (2009) Diabetes in Deutschland (DIG-Studie). A prospective 4-yearfollow-up study on the quality of treatment for type 2 diabetes in daily practice. Dtsch Med Wochenschr 134:291-297

109. Ramasamy R, Vannucci SJ, Yan SS et al (2005) Advanced glycation end products and RAGE: a common thread in aging, diabetes, neurodegeneration and inflammation. Glycobiology 15:16R-28R

127. Stratton IM, Cull CA, Adler Al et al (2006) Additive effects of glycaemia and blood pressure exposure on risk of complications in type 2 diabetes: a prospective observational study (UKPDS 75). Diabetologia 49:1761-1769

136. Wong TY, Mwamburi M, Klein R et al (2009) Rates of progression in diabetic retinopathy during different time periods: a systematic review and meta-analysis. Diabetes Care 32:2307-2313

Das vollständlige Literaturverzeichnis ...

... finden Sie in der html-Version dieses Beitrags im Online-Archiv auf der Zeitschriftenhomepage www.DerOphthalmologe.de 